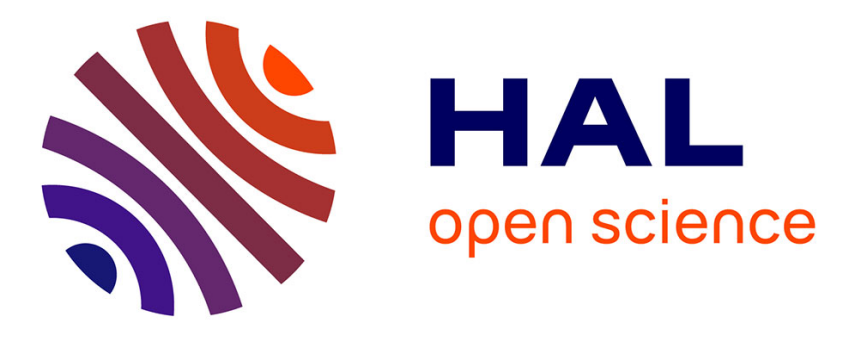

\title{
Transport of disturbance energy in hot and cold turbulent jets
}

\author{
Mohsen Talei, Michael J. Brear, Franck Nicoud, Daniel J Bodony, Alexis
}

Giauque

\section{- To cite this version:}

Mohsen Talei, Michael J. Brear, Franck Nicoud, Daniel J Bodony, Alexis Giauque. Transport of disturbance energy in hot and cold turbulent jets. 13th AIAA/CEAS Aeroacoustics Conference (28th AIAA Aeroacoustics Conference), May 2007, Rome, France. hal-02149294

\section{HAL Id: hal-02149294 \\ https://hal.science/hal-02149294}

Submitted on 6 Jun 2019

HAL is a multi-disciplinary open access archive for the deposit and dissemination of scientific research documents, whether they are published or not. The documents may come from teaching and research institutions in France or abroad, or from public or private research centers.
L'archive ouverte pluridisciplinaire HAL, est destinée au dépôt et à la diffusion de documents scientifiques de niveau recherche, publiés ou non, émanant des établissements d'enseignement et de recherche français ou étrangers, des laboratoires publics ou privés. 


\title{
Transport of disturbance energy in hot and cold turbulent jets
}

\author{
M. Talei*, M. J. Brear ${ }^{\dagger}$ \\ Department of Mechanical and Manufacturing Engineering, \\ University of Melbourne, Parkville 3010, Australia \\ F. Nicoud $\ddagger$ \\ Institut de Modélisation et de Mathématiques de Montpellier, \\ Université Montpellier II, Montpellier, France \\ D. J. Bodony ${ }^{\S}$ \\ Department of Aerospace Engineering, \\ University of Illinois at Urbana-Champaign, U. S. A. \\ A. Giauque \\ Center for Turbulence Research, Stanford University \\ U. S. A.
}

This paper presents a study of hot and cold turbulent jets by using the exact and linearised 'disturbance energy corollaries' proposed by Myers. Myers' energy corollaries are first extended to allow examination of results from Large Eddy Simulations (LES) around a turbulent base flow, via inclusion of additional disturbance energy flux and source terms. The budget of these extended forms of Myers' energy corollaries are then closed on selected LES results from Bodony \& Lele.

It is argued that Myers' exact disturbance energy flux should become the classical acoustic energy flux in the far-field surrounding a jet issuing into a quiescent domain. Thus, the volume integral of the exact disturbance energy source terms can be considered as related to the sources of far-field sound, although no information on the direction of the generated sound can be obtained using this approach. Moreover, this decomposition does not separate the radiating and non-radiating portions of the sources at a point in space. These claims are partially demonstrated in this paper, with closure of the disturbance energy budget showing that vortical, entropic and 'unresolved scale' source terms are the only significant source terms in this problem. For fixed acoustic Mach number above 0.7, the entropic source term dominates the vortical term for hot jets, which is in keeping with results from other studies on heated jets using acoustic analogies.

Nomenclature $\quad A \quad$ Surface area

a Sound velocity

$C_{p} \quad$ Specific heat at constant pressure

m Mass flux

$D \quad$ Jet diameter

$\mathbf{S}_{\mathbf{m}} \quad$ Sub grid scale terms for momentum equation $E \quad$ Total energy per unit mass

u Velocity

*m.talei@pgrad.unimelb.edu.au, Ph.D student, University of Melbourne, Australia

${ }_{\dagger}^{\dagger}$ mjbrear@unimelb.edu.au, Senior Lecturer, University of Melbourne, Australia

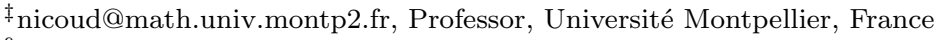

§bodony@uiuc.edu, Assistant Professor, University of Illinois at Urbana-Champaign, U. S. A.

『 Postdoctoral fellow, Stanford University, U. S. A. 


$\begin{array}{ll}F & \text { Unresolved LES quantity } \\ H & \text { Total enthalpy per unit mass } \\ k & \text { Thermal conductivity } \\ p & \text { pressure } \\ Q & \text { Conduction term } \\ q & \text { Heat conduction } \\ R & \text { Jet radius } \\ R e & \text { Reynolds number } \\ s & \text { Entropy } \\ S_{c} & \text { Sub grid scale terms for continuity equation } \\ S_{e} & \text { Sub grid scale terms for energy equation } \\ T & \text { Temperature } \\ t & \text { Time } \\ U_{j} & \text { Jet exit velocity } \\ V & \text { Volume } \\ x & \text { Cartesian coordinates } \\ S u b s c r i p t s \\ 0 & \text { Mean value } \\ 2 & \text { Second order value }\end{array}$

$\begin{array}{ll}\infty & \text { Ambient variable } \\ i & i^{t h} \text { coordinate variable } \\ j & \text { Jet variable } \\ r & \text { Radial component } \\ u n & \text { Unresolved value } \\ x & \text { Axial component } \\ \text { Symbols } \\ \boldsymbol{\psi} & \text { Viscosity term } \\ \boldsymbol{\xi} & \text { Vorticity } \\ \boldsymbol{\zeta} & \boldsymbol{\xi} \times \boldsymbol{u} \\ \gamma & \text { Ratio of specific heat } \\ \lambda & \text { Second coefficient of viscosity } \\ \mu & \text { Viscosity } \\ \rho & \text { Density } \\ \tau & \text { Viscosity stress tensor } \\ \text { Superscripts } \\ , & \text { Fluctuating value }\end{array}$

\section{Introduction}

\section{A. Hot and cold jets}

One of the more comprehensive experimental studies of the sound generated by cold and heated shock-free jets was presented by Tanna. ${ }^{1}$ Of note is the effect of heating the jet (figure 1), where Tanna found that the overall radiated sound intensity increased at all angles except $40^{\circ}$ if the jet's acoustic Mach number $U_{j} / a_{\infty}$ was less than 0.7. However, when the jet's acoustic Mach number was greater than 0.7, heating decreased the overall noise radiated to the far-field. This change in the effect of heating the jet on the overall intensity at an acoustic Mach number of roughly 0.7 is argued by some to still not be completely understood. ${ }^{2,3}$

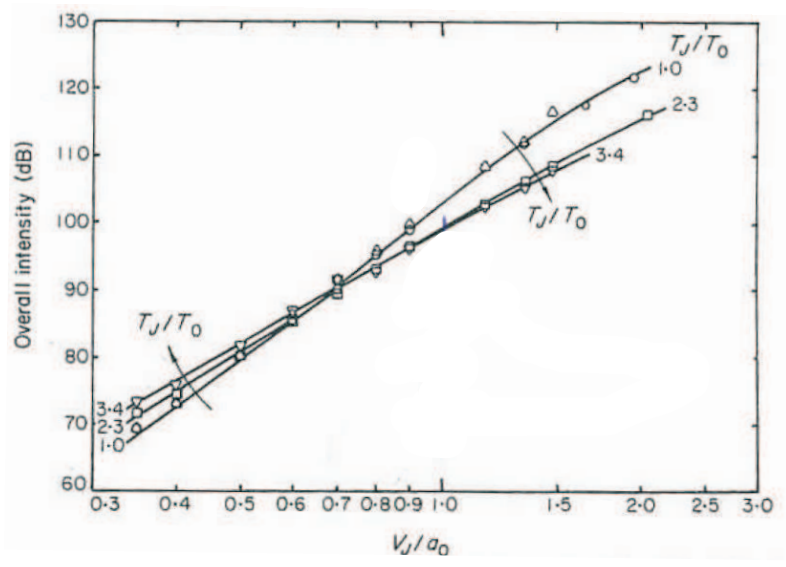

Figure 1. Measured overall intensity normal to jet axis $\left(\theta=90^{\circ}\right)$ for jets of different temperature ratios $\left(T_{j} / T_{\infty}\right)$ and acoustic Mach number $V_{j} / a_{\infty}$, see Tanna, ${ }^{1}\left(T_{j} / T_{\infty}\right): \triangle$, cold; o, $1.0 ; \square, 2.3 ; \nabla, 3.4$.

Prior to Tanna's experiments, the works of Tester \& Morfey ${ }^{4}$ and Mani ${ }^{5}$ used Lilley's analogy ${ }^{6}$ to argue for the existence of additional dipole source term due to temperature fluctuations. Mani further argued that there are additional jet noise scalings proportional to $M^{6}$ and $M^{4}$ as a result of density gradients in the mean flow. Furthermore, Goldstein ${ }^{7}$ derived an exact form of Lilley's equation with a velocity quadrupole and temperature dipole source terms. The appearance of an additional dipole for heated jets is not universally accepted. $^{8}$

Viswanathan ${ }^{3}$ presented an experimental study to show the importance of the jet Reynolds number on the noise radiated from hot jets. He argued that much of the experimental data may be contaminated 
by noise from valves, high flow velocities in ducts, sharp bends and other internal noise sources. Some of Tanna's work was reexamined, and for which there is some debate. ${ }^{3}$ Viswanathan discussed that there are changes in the jet behavior when the Reynolds number is lowered below a threshold, which he estimates to be $R e=\rho_{j} U_{j} D / \mu_{j} \approx 400,000$, when holding the jet temperature ratio and Mach number constant. Furthermore, he argued that changes in spectra at hot jets are not due to dipole sources for temperature.

Bodony \& Lele ${ }^{9}$ used large eddy simulations (LES) to predict the far-field noise for cold and hot jets. They used pressure data on a cylindrical Kirchhoff surface positioned at $5 D$ in the computational domain to relate the near-field to the far-field. Their results show reasonable agreement with the experimental data presented by Tanna ${ }^{1}$ for jets with $M_{j} \geq 0.9$. Further discussion of their results may be found in Bodony \& Lele. $^{10}$

\section{B. Disturbance and acoustic energies}

In arbitrary turbulent flows, any equation stating a form of energy conservation must start from the equations of motion that at least include non-zero mean flow quantities and entropy variation. ${ }^{11}$ It appears that only the energies defined by Morfey ${ }^{12}$ and Myers ${ }^{13}$ do this. In Morfey's analysis all terms containing entropy disturbances are shifted into the source term. However, in Myers' approach entropy disturbances remain in both the energy density and flux terms. Myers' equation was consistent with those developed earlier by $\mathrm{Chu}^{14}$ and Pierce ${ }^{15}$ for zero mean flow. Morfey ${ }^{12}$ adapted the definition of acoustic energy presented by Cantrell and Hart ${ }^{16}$ to extend the theory to sound generation in a non-uniform flow. He splits up the velocity field into rotational and irrotational parts, and then by using linearised equations of motion, the generalized acoustic energy equation was derived. Pierce ${ }^{15}$ derived a second order acoustic energy corollary based on linearised equations of motion for an homogenous and zero mean flow, however unlike the other analysis, entropy fluctuation appears as a part of the energy density and source terms in the energy corollary.

Myers ${ }^{13}$ derived exact and second order disturbance energy corollaries making use of mass, momentum, energy and entropy transport equation for an arbitrary steady flow. In Myers' equation, entropy disturbances appear in the energy density, flux and source terms. However, because the energy density and flux terms contained entropy disturbances, the resulting energies are not 'acoustic' and are properly called a more general 'disturbance energy'. ${ }^{13,14}$ For homentropic and irrotational flow, the linearised disturbance energy equation becomes the acoustic energy of Cantrell and Hart ${ }^{16}$ and for homogeneous and zero mean flow it becomes the energy corollary of Pierce. ${ }^{15}$ Unlike some of these previous works, the influence of mean flow on the acoustic waves propagating through the domain is considered in Myers' exact and second order equations. Furthermore, by looking at Myers' exact equation for turbulent flows, large disturbances can be modeled in nonlinear problems. ${ }^{11}$

To the authors' knowledge closure of Myers' disturbance energy equations on hot and cold jets has not been attempted previously. In this present work, the simulations of Bodony \& Lele ${ }^{9}$ for high-speed hot and cold jets are used.

\section{Theory}

The exact energy corollary derived by Myers is applicable to arbitrary steady flows. In order to apply Myers' equation to LES, it must first be derived for filtered data. Jet noise modeling also depends on the choice of base flow. Study of sound generation with respect to different kinds of base flow has been discussed by Goldstein. ${ }^{17}$ In the turbulent flows studied in this paper, the base flow is chosen to be the time averaged flow of the jet.

\section{A. Exact Energy Corollary}

The calculations were carried out using a non-dimensionalised form of the equations of motion in cylindrical coordinates $(r, \theta, x)$. The major quantities are non-dimensionalised by the far-field density $\rho_{\infty}$, velocity $a_{\infty}$, temperature $a_{\infty}^{2} / C_{p}$, axial and radial coordinates $R$, dynamic viscosity $\mu_{\infty}$ and thermal conductivity $k_{\infty}$. The jet Reynolds number is $R e=\rho_{j} U_{j} D / \mu_{j}$. The flow field variables are expressed as filtered equations of motion for an ideal gas,

$$
\frac{\partial \rho}{\partial t}+\frac{\partial \rho u_{j}}{\partial x_{j}}=S_{c},
$$




$$
\begin{gathered}
\frac{\partial \rho u_{i}}{\partial t}+\frac{\partial}{\partial x_{j}}\left(\rho u_{i} u_{j}+p \delta_{i j}-\tau_{i j}\right)=S_{m i} \\
\frac{\partial \rho E}{\partial t}+\frac{\partial}{\partial x_{j}}\left(\rho E+p u_{j}+q_{j}-\tau_{i j} u_{i}\right)=S_{e} .
\end{gathered}
$$

The filtered viscous stress $\tau_{i j}$ and heat flux $q_{i}$ are defined as

$$
\tau_{i j}=\mu\left(\frac{\partial u_{i}}{\partial x_{j}}+\frac{\partial u_{j}}{\partial x_{i}}\right)+\lambda \frac{\partial u_{k}}{\partial x_{k}} \delta_{i j}, \quad q_{i}=-k \frac{\partial T}{\partial x_{i}} .
$$

The sub-grid scale terms $S_{c}, \mathbf{S}_{\mathbf{m}}$ and $S_{e}$ are defined as

$$
\begin{gathered}
S_{c}=\frac{\partial F_{m j}}{\partial x_{j}} \\
S_{m i}=\frac{\partial F_{m i}}{\partial t}+\frac{\partial F_{u_{j} m_{i}}}{\partial x_{j}}, \\
S_{e}=\frac{\partial F_{\rho E}}{\partial t}+\frac{\partial F_{(\rho E+p) u_{j}}}{\partial x_{j}} .
\end{gathered}
$$

These terms were calculated in the same manner as by Bodony and Lele. ${ }^{9}$

The coefficients $\mu, \lambda$ and $k$ are evaluated using Sutherland's law. The Prandtl number is constant, $\operatorname{Pr}=0.71$. The filtered equation of state is used in its approximate form of

$$
p=(\gamma-1) \rho\left(E-\frac{1}{2} u_{i} u_{i}\right)
$$

The importance of unresolved scales in sound radiated from turbulent jets using LES has been discussed previously. ${ }^{18-20}$ To consider the effect of the unresolved scales in LES, Myers' equation is extended. To do this, we first need to derive new forms of the continuity, mass, momentum, entropy and energy equations on which Myers' equations are based. The equations of motion and entropy transport equation based on filtered quantities can then be written as

$$
\begin{gathered}
\frac{\partial \rho}{\partial t}+\nabla \cdot \mathbf{m}=S_{c} \\
\frac{\partial \mathbf{u}}{\partial t}+\boldsymbol{\zeta}+\boldsymbol{\nabla}\left(u^{2} / 2\right)+\nabla H-T \nabla s=\boldsymbol{\psi}+\boldsymbol{\psi}_{\boldsymbol{u n}}, \\
\frac{\partial \rho s}{\partial t}+\nabla \cdot(\rho \mathbf{u} s)=Q+Q_{u n}, \\
\frac{\partial}{\partial t}(\rho H-p)+\boldsymbol{\nabla} \cdot(\mathbf{m} H)-\mathbf{m} \cdot \boldsymbol{\psi}-T Q=\mathbf{m} \cdot \boldsymbol{\psi}_{\mathbf{u n}}+T Q_{u n}+\phi
\end{gathered}
$$

where

$$
\begin{gathered}
\boldsymbol{\psi}=\frac{1}{\rho} \frac{\partial \tau_{i j}}{\partial x_{j}}, \quad \boldsymbol{\psi}_{\mathbf{u n}}=\frac{\mathbf{S}_{\mathbf{m}}-\mathbf{u} S_{c}}{\rho} \\
Q=\frac{1}{T}\left(\tau_{i j} \frac{\partial u_{i}}{\partial x_{j}}-\frac{\partial q_{j}}{\partial x_{j}}\right), \quad Q_{u n}=\frac{1}{T}\left[S_{e}-\mathbf{u} \cdot \mathbf{S}_{\mathbf{m}}+\left(u^{2}-H+T s\right) S_{c}\right] \\
\phi=(H-T s) S_{c} .
\end{gathered}
$$

The parameters $\boldsymbol{\psi}_{\boldsymbol{u n}}, Q_{\text {un }}$ and $\phi$ express the effect of unresolved scales. The time average of equations 9 to 12 are

$$
\begin{gathered}
\boldsymbol{\nabla} \cdot \mathbf{m}_{\mathbf{0}}=S_{c 0} \\
\boldsymbol{\zeta}_{\mathbf{0}}+\nabla H_{0}-T_{0} \boldsymbol{\nabla} s_{0}-\left(T^{\prime} \nabla s^{\prime}\right)_{0}=\boldsymbol{\psi}_{\mathbf{0}}+\left(\boldsymbol{\psi}_{\boldsymbol{u n} \mathbf{n}}\right)_{\mathbf{0}}
\end{gathered}
$$




$$
\begin{gathered}
\boldsymbol{\nabla} \cdot\left(\mathbf{m}_{\mathbf{0}} s_{0}\right)+\boldsymbol{\nabla} \cdot\left(\mathbf{m}^{\prime} s^{\prime}\right)_{0}=Q_{0}+Q_{u n 0}, \\
\boldsymbol{\nabla} \cdot\left(\mathbf{m}_{\mathbf{0}} H_{0}\right)+\boldsymbol{\nabla} \cdot\left(\mathbf{m}^{\prime} H^{\prime}\right)_{0}-\mathbf{m}_{\mathbf{0}} \cdot \boldsymbol{\psi}_{\mathbf{0}}-\left(\mathbf{m}^{\prime} \cdot \boldsymbol{\psi}^{\prime}\right)_{0}-T_{0} Q_{0}-\left(T^{\prime} Q^{\prime}\right)_{0}=\phi_{0} \\
+\mathbf{m}_{\mathbf{0}} \cdot\left(\boldsymbol{\psi}_{\mathbf{u n}}\right)_{\mathbf{0}}+\left(\mathbf{m}^{\prime} \cdot \boldsymbol{\psi}_{\boldsymbol{u n}^{\prime}}{ }^{\prime}\right)_{0}+T_{0}\left(Q_{u n}\right)_{0}+\left(T^{\prime} Q_{u n}{ }^{\prime}\right)_{0} .
\end{gathered}
$$

Myers ${ }^{13}$ made use of the equations of mass, momentum, energy and entropy to derive an exact conservation equation for a 'fluctuating energy'. Here the same approach has been followed. By multiplying equation 9 by $\left(H_{0}-T_{0} s_{0}\right)$, equation 10 by $\mathbf{u}_{\mathbf{0}}$, equation 11 by $T_{0}$ and then subtracting these equations from equation 12 , after some algebra one can eventually obtain an exact disturbance energy equation for LES by also making use of equations 16 to 19

$$
\frac{\partial E}{\partial t}+\nabla \cdot \mathbf{W}=D
$$

The exact fluctuating energy density $E$ and flux vector $\mathbf{W}$ are

$$
E=\rho\left(H^{\prime}-T_{0} s^{\prime}\right)-\mathbf{m}_{\mathbf{0}} \cdot \mathbf{u}^{\prime}-p^{\prime}
$$

and

$$
\mathbf{W}=\mathbf{m}^{\prime}\left(H^{\prime}-T_{0} s^{\prime}\right)+\mathbf{m}_{\mathbf{0}} T^{\prime} s^{\prime}+\left(\mathbf{m}^{\prime} H^{\prime}\right)_{0}-T\left(\mathbf{m}^{\prime} s^{\prime}\right)_{0} .
$$

The exact source term D is

$$
D=D_{\xi}+D_{s}+D_{\psi}+D_{Q}+D_{u n}
$$

where

$$
\begin{aligned}
D_{\xi} & =\mathbf{m} \cdot \boldsymbol{\zeta}_{\mathbf{0}}+\mathbf{m}_{\mathbf{0}} \cdot \boldsymbol{\zeta} \\
D_{s} & =\mathbf{m}_{\mathbf{0}} s^{\prime} \boldsymbol{\nabla} T^{\prime}-\mathbf{m}^{\prime} s^{\prime} \boldsymbol{\nabla} T_{0}-\mathbf{m} \cdot\left(T^{\prime} \boldsymbol{\nabla} s^{\prime}\right)_{0}-\left(\mathbf{m}^{\prime} s^{\prime}\right)_{0} \boldsymbol{\nabla} T \\
D_{\psi} & =\mathbf{m}^{\prime} \cdot \boldsymbol{\psi}^{\prime}+\left(\mathbf{m}^{\prime} \cdot \boldsymbol{\psi}^{\prime}\right)_{0}, \\
D_{Q} & =T^{\prime} Q^{\prime}+\left(T^{\prime} Q^{\prime}\right)_{0} \\
D_{u n} & =(H-T s)^{\prime} S_{c}^{\prime}+\left[(H-T s)^{\prime} S_{c}^{\prime}\right]_{0}-\left(T^{\prime} s^{\prime}\right)_{0} S_{c} \\
& +T^{\prime} Q_{u n}{ }^{\prime}+\left(T^{\prime} Q_{u n}{ }^{\prime}\right)_{0} \\
& +\mathbf{m}^{\prime} \cdot \boldsymbol{\psi}_{\boldsymbol{u n}^{\prime}}{ }^{\prime}+\left(\mathbf{m}^{\prime} \cdot \boldsymbol{\psi}_{\text {un }}{ }^{\prime}\right)_{0} .
\end{aligned}
$$

The term $D_{\xi}$ in equation 23 is a source term due to vortical disturbances and $D_{s}$ is the source term due to entropic disturbances. In the case of homentropic flow, the entropic terms are zero and the first term can be considered as a source of acoustic energy if acoustic disturbances in $E$ and $\mathbf{W}$ are defined as irrotational. ${ }^{21}$ The terms $D_{\psi}$ and $D_{Q}$ represent viscous and thermal diffusion. The term $D_{u n}$ represents the unresolved scales where correlation between the filtered data and sub-grid scales is a significant part of the exact energy corollary.

Making use of the approximation $h^{\prime}=p^{\prime} / \rho_{0}+T_{0} s^{\prime}$ the second order flux term in the disturbance energy can then be derived,

$$
\begin{aligned}
\mathbf{W}_{\mathbf{2}} & =\left(p^{\prime}+\rho_{0} \mathbf{u}_{\mathbf{0}} \cdot \mathbf{u}^{\prime}\right)\left(\mathbf{u}^{\prime}+\frac{\rho^{\prime}}{\rho_{0}} \mathbf{u}_{\mathbf{0}}\right)+\mathbf{m}_{\mathbf{0}} T^{\prime} s^{\prime} \\
& +\left\{\left(\rho_{0} \mathbf{u}^{\prime}+\rho^{\prime} \mathbf{u}_{\mathbf{0}}\right)\left(\frac{p^{\prime}}{\rho_{0}}+T_{0} s^{\prime}+\mathbf{u}_{\mathbf{0}} \cdot \mathbf{u}^{\prime}\right)\right\}_{0}-T\left(\mathbf{m}^{\prime} s^{\prime}\right)_{0}
\end{aligned}
$$

For a statistically stationary flow, the time averaged form of the energy corollary can be used. By applying a time integral to the extended Myers' equation, the averaged form is obtained,

$$
\nabla \cdot \mathbf{W}_{\mathbf{0}}=D_{0}
$$


where

$$
\mathbf{W}_{\mathbf{0}}=2\left(\mathbf{m}^{\prime} H^{\prime}\right)_{0}-2 T_{0}\left(\mathbf{m}^{\prime} s^{\prime}\right)_{0}+\mathbf{m}_{\mathbf{0}}\left(T^{\prime} s^{\prime}\right)_{0}
$$

and

$$
\begin{aligned}
D_{0} & =2 \mathbf{m}_{\mathbf{0}} \cdot\left[\boldsymbol{\xi}_{\mathbf{0}} \times \mathbf{u}_{\mathbf{0}}+\left(\boldsymbol{\xi}^{\prime} \times \mathbf{u}^{\prime}\right)_{0}\right] \\
& +\mathbf{m}_{\mathbf{0}}\left(s^{\prime} \boldsymbol{\nabla} T^{\prime}-T^{\prime} \boldsymbol{\nabla} s^{\prime}\right)_{0}-2\left(\mathbf{m}^{\prime} s^{\prime}\right)_{0} \boldsymbol{\nabla} T_{0} \\
& +2\left(\mathbf{m}^{\prime} \cdot \boldsymbol{\psi}^{\prime}\right)_{0}+2\left(T^{\prime} Q^{\prime}\right)_{0} \\
& +2\left[(H-T s)^{\prime} S_{c}^{\prime}\right]_{0}-\left(T^{\prime} s^{\prime}\right)_{0} S_{c} \\
& +2\left(\mathbf{m}^{\prime} \cdot \boldsymbol{\psi}_{\text {un }}{ }^{\prime}\right)_{0} \\
& +2\left(T^{\prime} Q_{\text {un }}\right)_{0}
\end{aligned}
$$

Furthermore, time averaged form of the second order flux term is:

$$
\left(\mathbf{W}_{\mathbf{2}}\right)_{\mathbf{0}}=2\left[\left(p^{\prime}+\rho_{0} \mathbf{u}_{\mathbf{0}} \cdot \mathbf{u}^{\prime}\right)\left(\mathbf{u}^{\prime}+\frac{\rho^{\prime}}{\rho_{0}} \mathbf{u}_{\mathbf{0}}\right)\right]_{0}+\mathbf{m}_{\mathbf{0}}\left(T^{\prime} s^{\prime}\right)_{0}
$$

One can obtain the acoustic intensity in the far-field by making use of Myers' equation. By integrating equation 26 over the volume to the far-field and applying Gauss' theorem, it follows that

$$
\int_{S} \mathbf{W}_{\mathbf{0}} \cdot d \mathbf{A}=\int_{V} D_{0} d V
$$

The flux term $\mathbf{W}_{\mathbf{0}}$ in equation 30 becomes the classical acoustic energy flux $p^{\prime} u^{\prime}=p^{\prime 2} / \rho_{0} c_{0}$ in the far-field linear limit if the far-field is defined as having no entropy disturbances and zero mean velocity (disturbances entering the domain are known and can be accounted for in this flux balance). This should be a reasonable definition of the far-field since entropy disturbances should decay more quickly than sound. Under this assumption the disturbance energy balance in equation 30 becomes

$$
\int_{S}\left(2 p^{\prime} u^{\prime}\right)_{0} d A=\int_{S} \frac{2 p^{\prime 2}}{\rho_{0} c_{0}} d A=\int_{V} D_{0} d V
$$

It is noted that this final equation only gives the area and time averaged classical acoustic energy flux in the far-field and does not convey any information on the direction of the generated sound. Furthermore, it does not show where inside the domain the sound is generated. Equation 31 also expresses the contribution of exact source terms to sound radiated in far-field. This equation can therefore be used to explore possible mechanisms of sound generation by looking at the magnitude of each source term. However, this analysis does not separate the radiating and non-radiating components of the source terms at a point in space. In the case of high Reynolds number it is shown later that the major contributors to noise generation are the entropic and vortical terms. Viscosity and conduction terms whilst included in this work, are not important to the problem.

\section{Numerical methods}

\section{A. Computational Domain}

The cylindrical computational domain shown as the dashed box in figure 2 is used. ${ }^{9}$ The 'sponge' regions in the axial and radial direction are excluded from the computational field. In the radial direction, calculations are carried out up to $r / R=15$.

\section{B. Discrete approximations}

In order to minimise inconsistencies, the same spatial discretisation used by Bodony \& Lele ${ }^{9}$ is employed here. In the axial and radial directions, a compact finite difference scheme is utilised for first and second derivatives. $^{22}$ That is, 


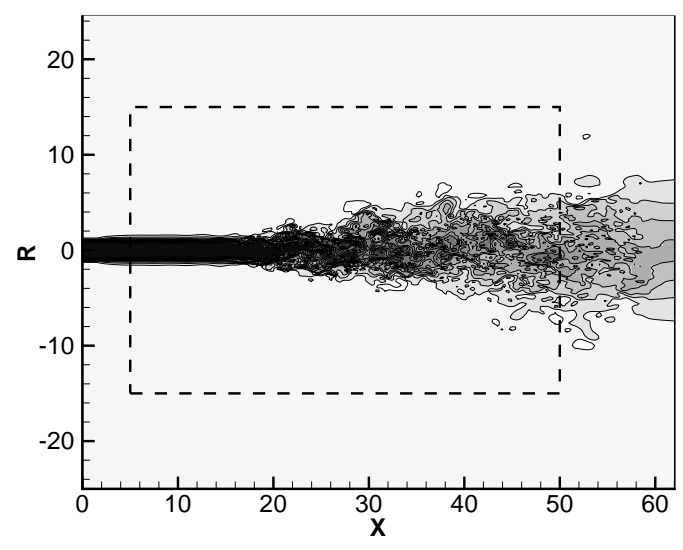

Figure 2. A slice thorough the computational domain

$$
\beta_{1}\left(f_{i-2}^{\prime}+f_{i+2}^{\prime}\right)+\alpha_{1}\left(f_{i-1}^{\prime}+f_{i+1}^{\prime}\right)+f_{i}^{\prime}=a_{1} \frac{f_{i+1}-f_{i-1}}{2 \Delta s}+b_{1} \frac{f_{i+2}-f i-2}{4 \Delta s}+c_{1} \frac{f_{i+3}-f_{i-3}}{6 \Delta s}
$$

for the first derivative and

$$
\beta_{2}\left(f_{i-2}^{\prime \prime}+f_{i+2}^{\prime \prime}\right)+\alpha_{2}\left(f_{i-1}^{\prime \prime}+f_{i+1}^{\prime \prime}\right)+f_{i}^{\prime \prime}=a_{2} \frac{f_{i+1}-2 f_{i}+f_{i-1}}{(\Delta s)^{2}}+b_{2} \frac{f_{i+2}-2 f_{i}+f_{i-2}}{4(\Delta s)^{2}}+c_{2} \frac{f_{i+3}-2 f_{i}-f_{i-3}}{9(\Delta s)^{2}}
$$

for the second derivative. The coefficients $\left\{\alpha_{i}, \beta_{i}, a_{i}, b_{i}, c_{i}\right\}_{i=1}^{2}$ found by Lui and Lele ${ }^{23}$ are used in this work. Circumferential derivatives are calculated using Fourier transforms and time derivatives are calculated using a forth order Pade scheme.

\section{Results and discussion}

The sets of cold and hot jet data presented earlier by Bodony \& Lele ${ }^{9}$ are used to evaluate the source and flux terms in Myers' equation extended to LES. The conditions of the simulations are given in table 1.

Table 1. Conditions of the simulations presented. The Nomenclature $\mathrm{spN}$, where $\mathrm{N}$ is an integer, refers to conditions tabulated in Tanna

\begin{tabular}{lccccc}
\hline $\mathrm{ID}$ & $U_{j} / a_{\infty}$ & $U_{j} / a_{j}$ & $T_{j} / T_{\infty}$ & $R e_{j}$ & $N_{r} \times N_{\theta} \times N_{x}$ \\
\hline \hline $\mathrm{sp} 7$ & 0.83 & 0.9 & 0.86 & 88000 & $128 \times 32 \times 256$ \\
$\mathrm{sp} 39$ & 1.47 & 0.97 & 2.30 & 84000 & $128 \times 32 \times 256$ \\
$\mathrm{sp} 621$ & 1.47 & 1.95 & 0.56 & 336000 & $128 \times 32 \times 256$ \\
\hline
\end{tabular}

Making use of equation 30, the integral of the source terms and flux terms over the cylindrical volume defined in figure 2 are calculated by summing the products of the exact source term at a given node with the exact elemental volume surrounding that node. As seen in table 2, the vorticity term has the largest magnitude of all source terms for the cold jet case, followed closely by the unresolved scale term $D_{\text {un }}$. The entropic terms are not significant relative to the magnitude of vorticity term. Furthermore, the entropy term has a negative sign which shows that cooling the jet increases the magnitude of entropy term and therefore reduces the sound pressure level. As expected, the magnitudes of the viscosity and conduction terms are very small at high Reynolds numbers.

Care must be taken when considering $D_{u n}$. As was discussed in section II, the approach followed in this paper can not determine what proportions of the disturbance energy source term at a point in space are radiating and non-radiating. A large source term at a point in space may actually be creating very little 
sound. It is only the volume integral of the source terms to the far-field that quantify the relative contribution of each source term in equation 28 to the far-field, area and time averaged, classical acoustic energy flux. Thus, in order to categorically determine the relative importance of any of the terms in the volume integral of equation 31, the axial extent of the computational domain must be long enough to allow the entropic disturbances and mean flow velocity to decay such that equation 31 is satisfied within the computational domain. As is shown later in this paper, this is not the case for the results presented in this paper. Further, the negative sign of the volume integral of $D_{u n}$ in table 2, like figures 3 to 5 discussed later, is consistent with the view that smaller turbulent scales dissipate energy generated by larger scales. This may suggest that non-radiating components of $D_{u n}$ at a point in space are significant. Further examination of all source terms on longer computational domains is therefore warranted.

\begin{tabular}{|c|c|c|c|}
\hline Source terms & sp62l & sp39 & $\mathrm{sp} 7$ \\
\hline $\int D_{\xi} d V$ & 10.958 & 1.344 & 1.278 \\
\hline $\int D_{s} d V$ & -0.325 & 5.872 & -0.025 \\
\hline $\int D_{u n} d V$ & -9.669 & -6.801 & -1.172 \\
\hline $\int D_{\psi} d V$ & -0.072 & -0.065 & -0.0296 \\
\hline $\int D_{Q} d V$ & -0.017 & -0.145 & -0.0019 \\
\hline $\int D d V$ & 0.875 & 0.205 & 0.0499 \\
\hline
\end{tabular}

Results of the hot jet data show a different source distribution (see sp39 in table 2). The main contributor to far-field sound in this case is now the entropic term. In particular, because of the lower density in the hot jet, comparison of cases sp621 and sp39 shows that the vortical term is smaller in this case and unlike the cold jets, the entropic term in the hot jet is positive. Increasing the temperature has two opposing effects on noise generation. On one hand, increasing the temperature lowers the mean density and therefore decreases the magnitude of the vortical term mainly due to the average mass flux $m_{0}$. On the other hand it increases the magnitude of the entropic term. However, the sum of the vortical and entropic source terms for the hot jet are less than the cold jet at the same acoustic Mach number. The effect of unresolved scales is similar to the cold jet and its order of magnitude does not change.

Table 3. Magnitude of flux terms in exact and second order disturbance energy equation

\begin{tabular}{lccc}
\hline Flux terms & sp62l & sp39 & sp7 \\
\hline \hline $\int W_{r} d A$ & 0.031 & 0.006 & 0.00016 \\
$\int W_{x} d A$ & 0.856 & 0.205 & 0.053 \\
$\int W_{r 2} d A$ & 0.031 & 0.0056 & 0.00010 \\
$\int W_{x 2} d A$ & 0.783 & 0.154 & 0.048 \\
$\int W d A$ & 0.887 & 0.211 & 0.053 \\
$\int W_{2} d A$ & 0.814 & 0.160 & 0.048 \\
\hline
\end{tabular}

Figures 3, 4 and 5 show contour plots of the time averaged source terms within the computational domain for all cases. The source terms are also averaged in the circumferential direction (and reflected in the $x$ axis). As seen in figure 3 the vorticity term is positive throughout most of the domain and the maximum value occurs inside the shear layer. Viscosity and conduction terms are very small compared to the other source terms. Contours of the unresolved source term shows a large area within the computational domain affected by small scales.

Figure 4 shows results for the hot jet that are qualitatively similar to those of the cold jet, but with the relative magnitude of each source term consistent with the volume integrated results in tables 2 and 3

Figure 6 shows a comparison between the exact and the second order disturbance energy flux and the 
(a)

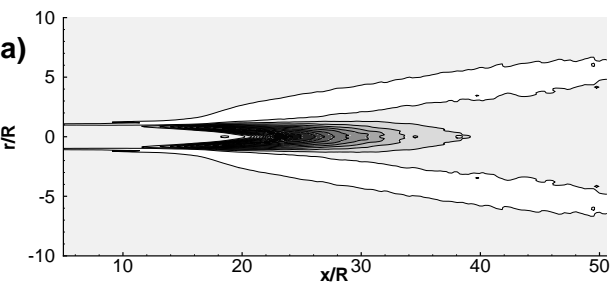

(c)

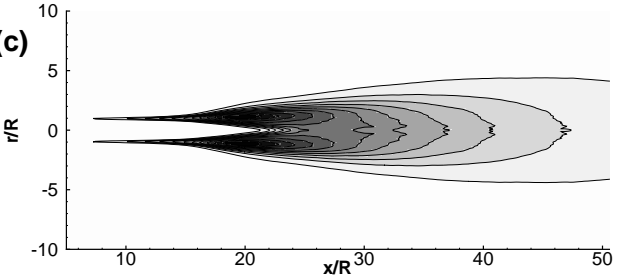

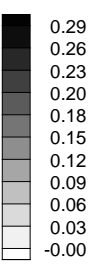

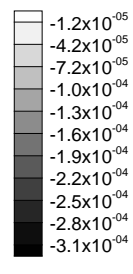

(b)
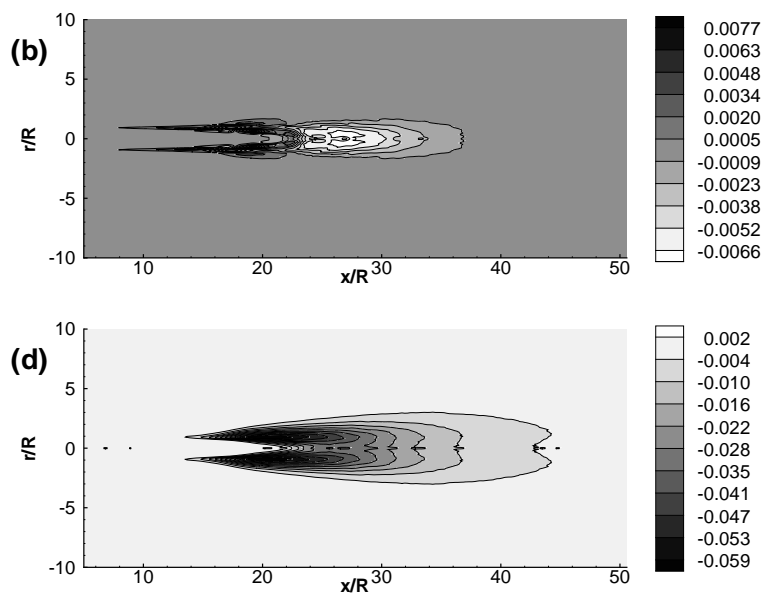

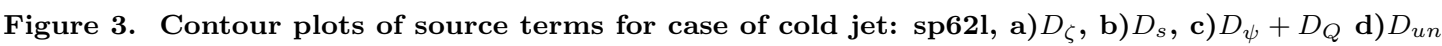

(a)

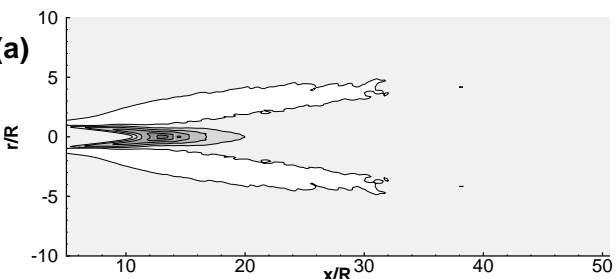

(c)

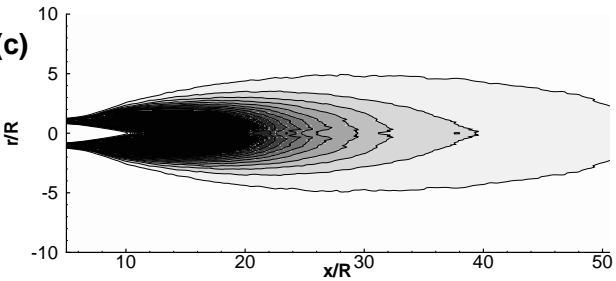

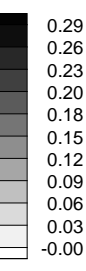

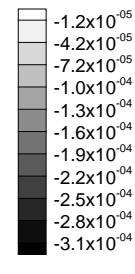

(b)
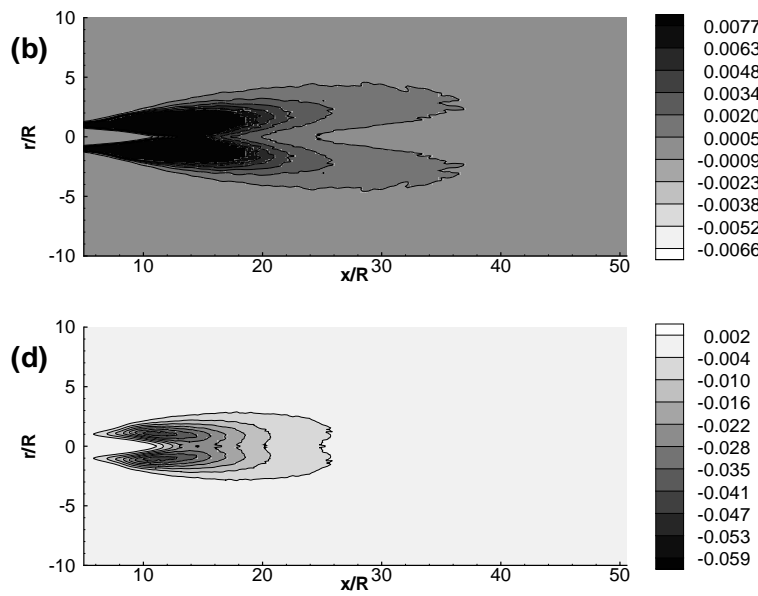

Figure 4. Contour plots of source terms for case of hot jet: sp39, a) $D_{\zeta}$, b) $\left.D_{s}, \mathbf{c}\right) D_{\psi}+D_{Q}$ d) $D_{\text {un }}$

(a)
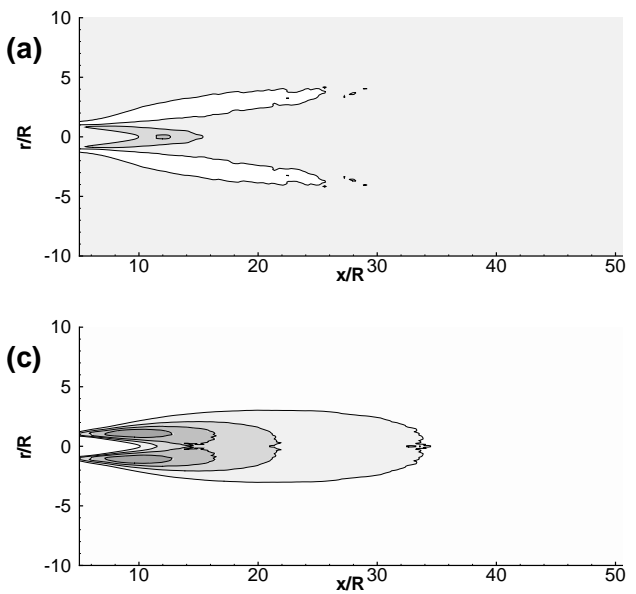
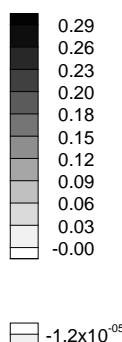

$1.2 \times 10^{-05}$ $-4.2 \times 10^{-05}$
$-7.2 \times 10^{-05}$

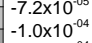
$-1.3 \times 10^{-04}$ $-1.6 \times 10^{-04}$ $-1.9 \times 10^{-04}$ $-2.2 \times 10^{-04}$ $2.5 \times 10^{-04}$ $-2.8 \times 10^{-04}$ $-3.1 \times 10^{-04}$ (b)
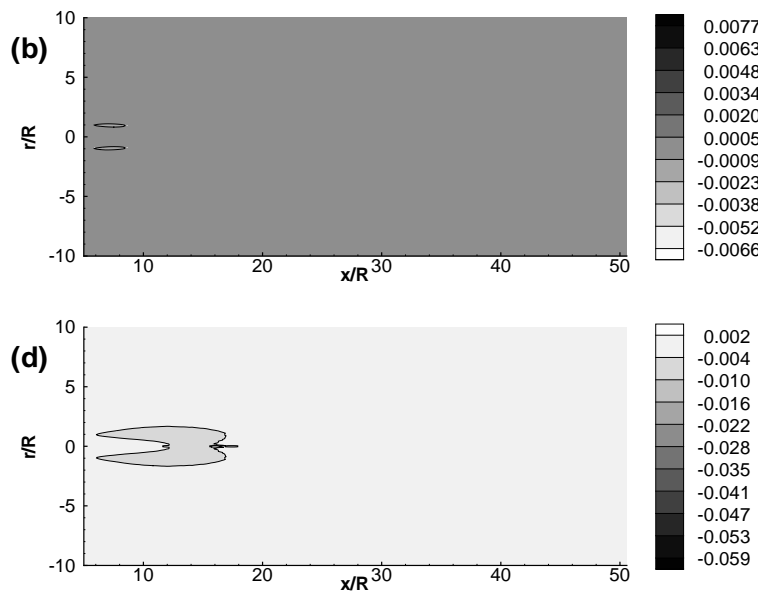

Figure 5. Contour plots of source terms for case of cold jet: sp7, a) $\left.\left.D_{\zeta}, \mathbf{b}\right) D_{s}, \mathbf{c}\right) D_{\psi}+D_{Q}$ d) $D_{u n}$ 
classical acoustic energy flux terms evaluated over a cylindrical surface extending from $x / R=5$ to $x / R=50$ and with varying radius. The quantity plotted is the respective flux multiplied by the area of the cylindrical surface. For all jets the second order disturbance energy flux term follows the exact flux term reasonably closely. All graphs show that by $r / R=15$, the three fluxes are effectively the same and the previously defined far-field has been reached in the radial direction. This means that entropic disturbances are insignificant by $r / R=15$, which is not a surprising result for this problem, given the flow structure. Of course within the domain the classical acoustic energy flux has uncertain physical meaning until it reaches the far-field since the mean flow can be inhomogeneous and the disturbances nonlinear.
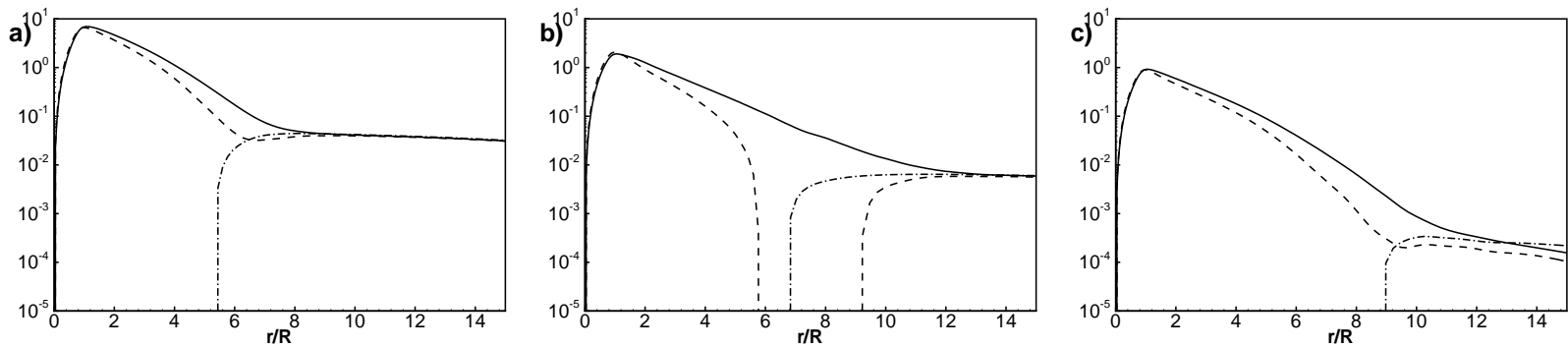

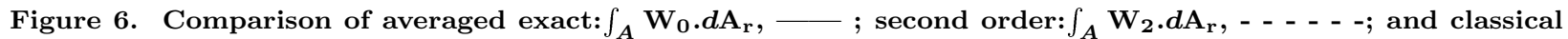
acoustic energy flux: $\int_{A}\left(2 p^{\prime} u^{\prime}\right)_{0} . d A_{r},-. .-. . . .-. .-;$ for a) cold $(\operatorname{sp62l})$, b) hot $(\operatorname{sp39})$, and c) cold (sp7) jet
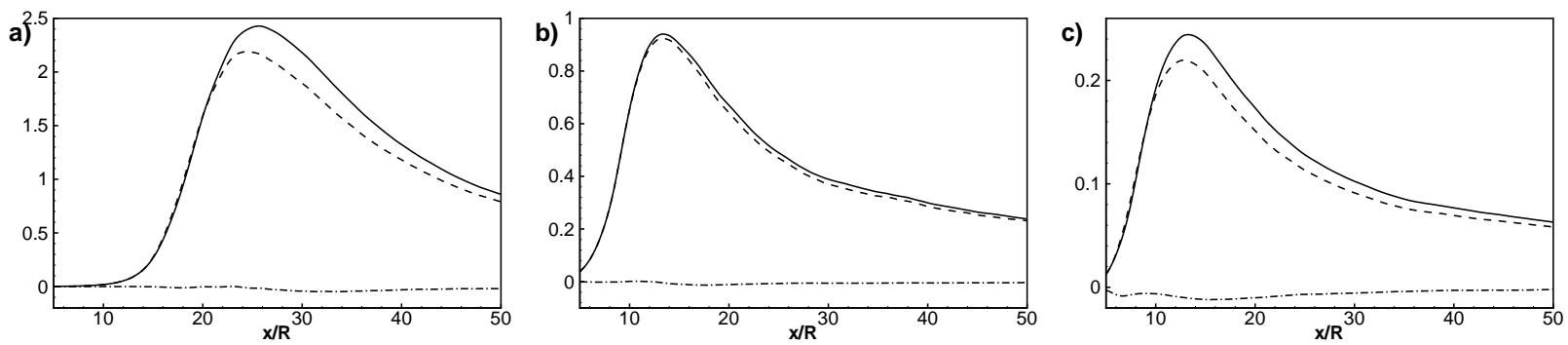

Figure 7. Comparison of averaged exact: $\int_{A} \mathrm{~W}_{0} \cdot d \mathrm{~A}_{\mathrm{x}},-$; second order: $\int_{A} \mathrm{~W}_{2} . d \mathrm{~A}_{\mathrm{x}},-\cdots---;$ and classical acoustic energy flux: $\int_{A}\left(2 p^{\prime} \mathbf{u}^{\prime}\right)_{0} . d \mathrm{~A}_{\mathrm{x}},-. .-\ldots-. . .-$; for a) cold $(\mathrm{sp62l})$, b) hot (sp39), and c) cold (sp7) jet

In figure 7 the axial flux terms for all jets are shown. Unlike the radial flux there is a large difference between exact and classical acoustic energy flux terms due to the effect of entropy waves coming out of the domain. Furthermore, there is a relatively small difference between second order term and the exact flux term within the computational domain. Figure 7 therefore shows that the simulations used to be of substantially long axial extent in order for these entropy waves to decay. Nonetheless, this should not affect the sound generation problem, since figures 3 to 5 show that the significant regions of all source terms are already captured.

\section{Conclusion}

This paper presented an investigation of hot and cold turbulent jets using an extended form of the exact and linearised 'disturbance energy corollaries' proposed by Myers. ${ }^{13}$ The extended forms of Myers' energy corollaries allow examination of results from Large Eddy Simulations (LES) around a turbulent base flow, via inclusion of additional disturbance energy flux and source terms. The budget of these disturbance energy corollaries were then closed on selected LES results from Bodony \& Lele ${ }^{9}$ which, in turn, are simulations of cases studied in the earlier experiments of Tanna. ${ }^{1}$

It was argued that Myers' exact disturbance energy flux should become the classical acoustic energy flux in the far-field once i) entropic disturbances become negligible, ii) all disturbances are linear and iii) the mean flow is stationary. This should be reasonable since entropy disturbances should decay much more quickly than sound waves. If this is so, for a sufficiently long averaging period, the classical acoustic energy 
flux averaged over the far-field boundary should equal the volume integral of the exact source terms within the boundary. Thus, volume and time integral of the exact, disturbance energy source terms within the domain can be considered as sources of the area and time averaged, far-field sound, although no information on the direction of the far-field sound and the spatial location of the sound source within the volume can be obtained using this approach. These claims were partially demonstrated in this paper, with the radial disturbance energy flux becoming very close to the classical acoustic energy flux within the computational domain in all cases studied. However, whilst the region of significant disturbance energy source terms is captured in all cases, the axial extent of the computational domain in Bodony \& Lele's ${ }^{9}$ simulations did not allow sufficient decay of convected entropy disturbances.

Closure of the disturbance energy budget nonetheless showed that vortical, entropic and 'unresolved scale' source terms are the only significant source terms in this problem. For fixed acoustic Mach number above 0.7 , the entropic source term dominates the vortical term for hot jets, which is in keeping with results from other studies on heated jets using acoustic analogies.

\section{Acknowledgements}

This work commenced while the second and later authors were attending the Center for Turbulence Research (CTR) Summer Program at Stanford University in July - August, 2006. The support of Stanford University is gratefully acknowledged.

\section{References}

\footnotetext{
${ }^{1}$ Tanna, H. K., "An experimental study of jet noise Part I: Turbulent mixing noise," J. Sound Vib, Vol. 50, No. 3, 1977, pp. $405-428$.

${ }^{2}$ Huff, D., "Technical Report NASA CP-2001-211152," Proceedings of the jet noise workshop, NASA, Cleveland, Ohio, 2001.

${ }^{3}$ Viswanthan, K., "Aeroacoustics of hot jets," J. Fluid Mech., Vol. 516, 2004, pp. 39-82.

${ }^{4}$ Tester, B. J. and Morfey, C. L., "Developments in jet noise modelling - theoretical predictions and comparisons with measured data," J. Sound Vib., Vol. 46, No. 1, 1971, pp. 79-103.

${ }^{5}$ Mani, R., "The influence of jet flow on jet noise. Part 2. The noise of heated jets," J. Fluid Mech., Vol. 73, No. 04, 1976, pp. $779-793$.

${ }^{6}$ Lilley, G. M., "On the noise from air jets," $A G A R D$, Vol. CP 131, 1971.

${ }^{7}$ Goldstein, M. E., "An exact form of Lilley's equation with a velocity quadrupole/temperature dipole source term," $J$. Fluid Mech., Vol. 443, 2001, pp. 231-236.

${ }^{8}$ Tam, C. and Auriault, L., "Jet Mixing Noise from Fine-Scale Turbulence," AIAA Journal, Vol. 37, No. 2, 1999, pp. 145153.

${ }^{9}$ Bodony, D. J. and Lele, S. K., "On using large-eddy simulation for the prediction of noise from cold and heated turbulent jets," Physics of Fluids, Vol. 17, 2005, pp. 085103.

${ }^{10}$ Bodony, D. J. and Lele, S. K., "Review of the current status of jet noise predictions using large-eddy simulation," 44 th AIAA Aerospace Sciences Meeting and Exhibit, 2006, pp. 1-36.

${ }^{11}$ Giauque, A., Poinsot, T., Brear, M., and Nicoud, F., "Budget of disturbance energy in gaseous reacting flows," Proceedings of the Summer Program, Center for Turbulence Research, NASA AMES, Stanford University, USA, 2006, pp. $285-297$.

${ }^{12}$ Morfey, C. L., "Acoustic energy in non-uniform flows," J. Sound Vib., Vol. 14, No. 2, 1971, pp. 159-170.

${ }^{13}$ Myers, M. K., "Transport of energy by disturbances in artitrary steady flows," J. Fluid Mech., Vol. 226, 1991, pp. 383-400.

${ }^{14} \mathrm{Chu}$, B. T., "On the energy transfer to small disturbances in fluid flow (Part 1)," ACTA Mechanica, Vol. 1, 1965, pp. 215-234.

${ }^{15}$ Pierce, A. D., Acoustics - An Introduction to its Physical Principles and Applications, McGraw-Hill, 1981.

${ }^{16}$ Cantrell, R. H. and Hart, R. W., "Interaction between sound and flow in acoustic cavities: mass, momentum and energy considerations," Journal of the Acoustical Society of America, Vol. 36, 1964, pp. 697-706.

${ }^{17}$ Goldstein, M. E., "A generalized acoustic analogy," J. Fluid Mech., Vol. 488, 2003, pp. 315-333.

${ }^{18}$ Seror, C., Sagaut, P., Bailly, C., and Juve, D., "On the radiated noise computed by large-eddy simulation," Phys. Flui., Vol. 13, No. 2, 2001, pp. 476-487.

${ }^{19}$ Bodony, D. J. and Lele, S. K., "Spatial scales decomposition of shear layer turbulence and the sound sources associated with the missing scales in a large-eddy simulation," $8^{\text {th }}$ AIAA Aeroacoustics Conference, Breckenridge,CO, 17-19 Jun 2002, pp. $1-13$.

${ }^{20}$ Bodony, D. J. and Lele, S. K., "A statistical subgrid scale noise model: formulation," $9^{\text {th }}$ AIAA Aeroacoustics Conference, Hilton Head, SC, 12-14 May 2003.

${ }^{21}$ Dowling, A. P., "Acoustics of unstable flows," Theoretical and Applied Mechanics, 1997, pp. 171-186.

${ }^{22}$ Lele, S. K., "Compact Finite Diference Schemes with Spectral-like Resolution," J. Comp. Phys., Vol. 103, 1992, pp. 16-42.

${ }^{23}$ Lui, C. and Lele, S. K., "Direct Numerical Simulation of Spatially Developing, Compressible,Turbulent Mixing Layers," $39^{\text {th }}$ AIAA Aerospace Sciences Meeting and Exhibition, Reno,Nevada, 14-17 Jan 2001.
} 